\title{
Genome-wide gene expression profiling of the Angelman syndrome mice with Ube3a mutation
}

\author{
Daren Low ${ }^{1}$ and Ken-Shiung Chen ${ }^{\star 1}$
}

Angelman syndrome (AS) is a human neurological disorder caused by lack of maternal UBE3A expression in the brain. UBE3A is known to function as both an ubiquitin-protein ligase (E3) and a coactivator for steroid receptors. Many ubiquitin targets, as well as interacting partners, of UBE3A have been identified. However, the pathogenesis of AS, and how deficiency of maternal UBE3A can upset cellular homeostasis, remains vague. In this study, we performed a genome-wide microarray analysis on the maternal Ube3a-deficient (Ube3a ${ }^{\mathrm{m}-/ \mathrm{p}+}$ ) AS mouse to search for genes affected in the absence of Ube $3 a$. We observed 64 differentially expressed transcripts ( 7 upregulated and 57 downregulated) showing more than 1.5-fold differences in expression $(P<0.05)$. Pathway analysis shows that these genes are implicated in three major networks associated with cell signaling, nervous system development and cell death. Using quantitative reverse-transcription PCR, we validated the differential expression of genes ( $F g f 7$, Glra1, Mc1r, Nr4a2, SIc5a7 and Epha6) that show functional relevance to AS phenotype. We also show that the protein level of melanocortin 1 receptor (Mc1r) and nuclear receptor subfamily 4, group A, member 2 (Nr4a2) in the AS mice cerebellum is decreased relative to that of the wild-type mice. Consistent with this finding, expression of small-interfering RNA that targets Ube3a in P19 cells caused downregulation of Mc1r and Nr4a2, whereas overexpression of Ube3a results in the upregulation of $\mathrm{Mc} 1 \mathrm{r}$ and $\mathrm{Nr} 4 \mathrm{a} 2$. These observation help in providing insights into the genesis of neurodevelopmental phenotype of AS and highlight specific area for future research. European Journal of Human Genetics (2010) 18, 1228-1235; doi:10.1038/ejhg.2010.95; published online 23 June 2010

Keywords: E6AP; neurological disorder; Mc1r; Nr4a2

\section{INTRODUCTION}

Angelman syndrome (AS, OMIM 105830) is a human neurogenetic disorder affecting approximately 1 in 10000-40 000 newborns. ${ }^{1,2}$ AS patients show distinct dysmorphic facial features, inappropriate laughter, ataxia and motor dysfunction. ${ }^{3}$ Many patients also show seizures of variable magnitude, learning deficit and hypopigmentation. ${ }^{3}$ Molecular genetic studies show that AS is caused by the lack of functional UBE3A expression. ${ }^{4}$ UBE3A encodes the E6-AP ubiquitin ligase, and is found to be imprinted in a selected brain cell population such that only the maternal inherited allele is expressed. ${ }^{5}$ UBE3A functions mainly through targeting proteins for proteosomal degradation, and is also known to function as a coactivator for steroid hormone receptors. ${ }^{6,7}$

In the brain neurons in which $U B E 3 A$ is imprinted, it was found that $U B E 3 A$ is localized in the nucleus, presynaptic and postsynaptic regions. ${ }^{6,8}$ Ube3a-deficient mice showed long-term potentiation impairment and abnormal dendritic spine number and morphology. ${ }^{8,9}$ Similarly, in Drosophila, either deficiency or overexpression of dUBE3A leads to reduced dendritic branching. ${ }^{10}$ These reports suggested that UBE3A may exert its effect locally in the synaptic region regulating dendritic spine development, synaptic plasticity and functions. Ubiquitination targets of UBE3A, such as p53, epithelial cell-transforming sequence 2 oncogene (Ect2) and tuberous sclerosis 2 (TSC2), and interacting partners, such as HSP70, have been identified. ${ }^{6,11-14}$ However, the role of UBE3A in the brain and how its deficiency can result in AS remain unclear.

To find out which genes are affected in the absence of functional Ube3a in the brain, we performed a genome-wide microarray expression analysis on wild-type and $U b e 3 a^{\mathrm{m}-/ \mathrm{p}+}$ mice. We identified 64 genes showing greater than 1.5-fold differences in expression $(P<0.05)$ in the Ube $3 a^{\mathrm{m}-/ \mathrm{p}+}$ mouse. Pathway analysis reveals that they are involved in three major networks, including cell signaling, nervous system development and cell death. Expression of functionally relevant candidates from each pathway was validated using quantitative reverse-transcription PCR (qRT-PCR). Among them, both melanocortin 1 receptor (Mc1r), which is shown to have a neuroprotective effect in the brain, and nuclear receptor subfamily 4 , group A, member 2 (Nr4a2), which is critical for survival of dopamine neurons and whose expression can be induced by Mclr, are downregulated. ${ }^{15-17}$ We have confirmed that the decline of $\mathrm{Mclr}$ and Nr4a2 at transcriptional level is reflected at the protein level in the Ube $3 a^{\mathrm{m}-/ \mathrm{p}+}$ mouse. Using RNA interfering approach, we have further shown that shRNA-mediated knockdown of Ube3a in P19 cells leads to downregulation of Mc1r and Nr4a2. In contrast, overexpression of Ube3a in P19 cells results in the upregulation of Mc1r and Nr4a2 mRNA levels. These results provide informative molecular insights on the pathogenesis of AS, as well as the functions of Ube3a.

\section{MATERIALS AND METHODS}

\section{Ethics statement/mouse strains}

All animal work was maintained, performed and approved by the School of Biological Sciences Animal Facility based on guidelines from the Institutional Animal Care and Use Committee. Ube $3 a^{\mathrm{m}-/ \mathrm{p}+}$ mice were generated and confirmed using PCR as previously described. ${ }^{9}$ 


\section{Microarray sample preparation and hybridization}

Cerebellum total RNA were extracted from 6-week-old female wild-type and Ube $3 a^{\mathrm{m}-/ \mathrm{p}+}$ mice using Trizol reagents (Invitrogen Inc., Carlsbad, CA, USA) according to the manufacturer's protocol. Starting total RNA ( $1 \mu \mathrm{g})$ was used from each sample for the Affymetrix GeneChip Mouse Exon Array analysis. Samples were processed according to Affymetrix GeneChip Whole Transcript Sense Target Labeling Assay (Affymetrix Inc., Santa Clara, CA, USA).

\section{Analysis and statistic}

Four biological replicates from each of wild-type and $U b e 3 a^{\mathrm{m}-/ \mathrm{p}+}$ mice were included in the final analysis to detect differential gene expression derived from Partek Genomic Suite (Partek Inc., St Louis, MO, USA). The Affymetrix generated CEL files containing raw data were subjected to RMA normalization, background subtraction and summarization. One-way ANOVA was subsequently performed to detect $P$-values for the respective gene expression fold changes. The criteria for a gene to be considered differentially expressed were set at $P \leq 0.05$ and a minimal fold change of 1.5 -fold. Gene ontology and network/pathway analyses were performed using Ingenuity Pathways Analysis software (Ingenuity System, Redwood City, CA, USA). Quality control analysis was performed using Affymetrix Expression Console software with reference to Affymetrix quality control white papers.

\section{Semiquantitative reverse-transcription PCR}

Total RNA $(2 \mu \mathrm{g})$ from each sample was treated with RQ1 DNase (Promega, Madison, WI, USA) and then reverse-transcribed using Superscript (Invitrogen Inc.). Subsequently, $1 \mu \mathrm{l}$ of the cDNA template generated was used for each PCR reaction using Faststart Taq Polymerase (Roche, Indianapolis, IN, USA), and cycling conditions were $95^{\circ} \mathrm{C}$ for $10 \mathrm{~min}$, then $30-35$ cycles of $30 \mathrm{~s}$ at $95^{\circ} \mathrm{C}$, $30 \mathrm{~s}$ at $60^{\circ} \mathrm{C}$ and $30 \mathrm{~s}$ at $72^{\circ} \mathrm{C}$, followed by a final extension of $10 \mathrm{~min}$ at $72^{\circ} \mathrm{C}$.

\section{qRT-PCR}

A $25 \mu \mathrm{l}$ reaction containing iTaq SYBR Green Supermix with ROX (Bio-Rad, Hercules, CA, USA) was prepared according to the manufacturer's instructions, and PCR-amplified using ABI 7500 System (Applied Biosystems, Carlsbad, CA, USA). Cycling conditions include $10 \mathrm{~min}$ at $50^{\circ} \mathrm{C}, 10 \mathrm{~min}$ at $95^{\circ} \mathrm{C}$, then 45 cycles of $30 \mathrm{~s}$ at $95^{\circ} \mathrm{C}, 30 \mathrm{~s}$ at $60^{\circ} \mathrm{C}$ and $32 \mathrm{~s}$ at $72^{\circ} \mathrm{C}$. The experiment was performed in three biological replicates. The $C_{\mathrm{T}}$ value for each gene was determined in the linear phase of the amplification for each gene, and normalized to $C_{\mathrm{T}}$ value of G3pdh to obtain the $\Delta C_{\mathrm{T}}$. The fold change for each gene was obtained using $2^{-(\text {mean wild-type } \Delta C \mathrm{~T} \text {-mean } U b e 3 a(\mathrm{~m}-/ \mathrm{p}+) \Delta C \mathrm{~T})}$. A simple $t$-test was performed on the $\Delta C_{\mathrm{T}}$ for each gene to obtain a $P$-value for differential expression

\section{Western blot}

Cerebellum from six-week-old female mouse was homogenized in lysis buffer ( $30 \mathrm{~mm}$ Tris, $7 \mathrm{~m}$ urea, $2 \mathrm{~m}$ thiourea, $4 \%$ CHAPS ( $\mathrm{pH} 8.5$ ), protease inhibitor). P19 cells were lysed in lysis buffer (0.1 м Tris- $\mathrm{HCl}$ ( $\mathrm{pH} 7.4), 150 \mathrm{~mm} \mathrm{NaCl}, 1 \mathrm{~mm}$ EDTA, $2 \mathrm{~mm} \mathrm{MgCl}_{2}, 1 \%$ Triton X-100, $15 \%$ glycerol, $2 \mathrm{mg} / \mathrm{ml}$ phenylmethylsulfonyl fluoride and protease inhibitor). Total protein $(10 \mu \mathrm{g})$ was separated in SDS-PAGE, and Western blotting was performed using primary anti-Ube3a (Bethyl Laboratories, Montgomery, TX, USA), anti-Mclr and anti-Nr4a2 antibody (Santa Cruz Biotechnology, Santa Cruz, CA, USA), and detected by chemiluminescence horseradish-peroxidase-conjugated secondary antibody.

\section{shRNA expressing vector construct}

A 19-mer ( $5^{\prime}$-ctt-cgt-atg-gat-aac-aat-g-3') against exon 5 of Ube3a (GenBank accession number: NM_011668.2) was cloned into pSUPER.puro vector (Oligoengine, Seattle, WA, USA) for the shRNA-mediated Ube3a knockdown. The targeted exon 5 corresponds to the exon 2 that was deleted in the mouse previously described. ${ }^{9}$ This construct will be referred to as pUbe3aKD hereafter.

\section{Ube3a overexpression vector construct}

Ube3a coding region was amplified from p3003 pGEM E6-AP (Addgene, Cambridge, MA, USA) using forward primer: 5 '-gat-cta-ggt-acc-tat-ggc-cacagc-ttg-taa-aag-3' and reverse primer: $5^{\prime}$-act-gat-gga-tcc-tta-cag-cat-gcc-aaatcc- $3^{\prime}$. The $2559 \mathrm{bp}$ PCR product was then cloned into pcDNA/HisMaxB vector (Invitrogen Inc.) between the KpnI and BamHI restriction sites. To track the transfection/expression efficiency, we amplified a $1368 \mathrm{bp}$ fragment containing the internal ribosomal entry site-eGFP fusion from pIGCN21 vector (NCI-Frederick, Frederick, MD, USA) using forward primer: $5^{\prime}$-gatcta-gga-tcc-gcc-aag-cta-tcg-aat-tcc-gc- $3^{\prime}$ and reverse primer: $5^{\prime}$-act-gat-gcg-gccgct-tat-gca-gaa-ttc-gaa-gct-tga-gc-3' and cloned between the BamHI and NotI sites of the pcDNA/HisMaxB vector. This vector will be referred to as pUbe3aOE hereafter.

\section{Cell culture and transfection}

P19 cells were cultured in $\alpha$-minimum essential medium supplemented with $7.5 \%$ bovine calf serum and $2.5 \%$ fetal bovine serum. P19 cells $\left(4 \times 10^{5}\right)$ were transfected with pUbe3aKD or pUbe3aOE in a six-well plate using Lipofectamine 2000 reagent (Invitrogen Inc.) according to the manufacturer's protocol. Cells were collected $24 \mathrm{~h}$ later.

\section{RESULTS}

\section{Whole-genome microarray analysis}

We are interested in identifying genes that are affected in the absence of functional Ube3a. Because Ube3a is expressed from the maternal inherited allele in the cerebellum, ${ }^{5}$ we checked for differential gene expression between wild-type and Ube $3 \mathrm{a}^{\mathrm{m}-/ \mathrm{p}+}$ mice. We performed microarray analysis using Affymetrix GeneChip Mouse Exon array on four biological replicates from each group. Genes are considered to be differentially expressed if they show a fold change of at least 1.5-fold and $P \leq 0.05$.

We analyzed the gene expression profiles between the two groups using the Core Probeset, which is based on highly confident supporting evidence from RefSeq and GenBank full-length mRNAs. This yielded a total of 64 differentially expressed genes ( 7 upregulated and 57 downregulated) that were statistically significant (Table 1).

Table 1 Microarray gene expression analysis (Core Probeset): wild-type $\left(U b e 3 a^{m+/ p+}\right)$ vs Ube $3 a$ knockout $\left(U b e 3 a^{m-/ p+}\right)$ mice

\begin{tabular}{|c|c|c|c|c|c|c|}
\hline No. & Affymetrix transcript ID & NCBI accession no. & Gene symbol & Gene name & P-value & Fold change \\
\hline \multicolumn{7}{|c|}{ Upregulation in mutant } \\
\hline 1 & 6880900 & NM_008008 & Fgf7 & Fibroblast growth factor $7 /$ & 0.022 & 2.091 \\
\hline 2 & 6927341 & NM_080445 & B3galt6 & UDP-Gal: $\beta$ Gal $\beta$ 1,3-galactosyltransferase & 0.036 & 1.907 \\
\hline 3 & 6817951 & AK029771 & 9330180L21Rik & RIKEN cDNA 9330180L21 & 0.015 & 1.750 \\
\hline 4 & 6985703 & NM_026758 & Mphosph6 & M-phase phosphoprotein 6 & 0.039 & 1.701 \\
\hline 5 & 6786954 & AK085965 & 2010316F05Rik & RIKEN cDNA $2010316 F 05$ & 0.027 & 1.587 \\
\hline 6 & 6935555 & NM_019647 & $R p / 21$ & Ribosomal protein L21 & 0.032 & 1.542 \\
\hline 7 & 6935197 & NM_001038703 & Gpr146 & G-protein-coupled receptor 146 & 0.035 & 1.541 \\
\hline \multicolumn{7}{|c|}{ Downregulation in mutant } \\
\hline 8 & 6992946 & NM_178676 & Entpd3 & Ectonucleoside triphosphate diphosphohydrolase 3 & 0.008 & -1.501 \\
\hline 9 & 6976233 & NM_080438 & Glra3 & Glycine receptor, $\alpha-3$ subunit & 0.045 & -1.502 \\
\hline
\end{tabular}


Table 1 (Continued)

\begin{tabular}{|c|c|c|c|c|c|c|}
\hline No. & Affymetrix transcript ID & NCBI accession no. & Gene symbol & Gene name & P-value & Fold change \\
\hline 11 & 6963197 & NM_007627 & Cckbr & Cholecystokinin B receptor & 0.012 & -1.517 \\
\hline 13 & 6898010 & NM_008604 & Mme & Membrane metallo-endopeptidase & 0.027 & -1.532 \\
\hline 14 & 6925872 & NM_008154 & Gpr3 & G-protein-coupled receptor 3 & 0.029 & -1.542 \\
\hline 15 & 6846576 & NM_007938 & Epha6 & Eph receptor A6 & 0.004 & -1.555 \\
\hline 18 & 6870979 & BC023699 & Al790298 & Expressed sequence Al790298 & 0.024 & -1.566 \\
\hline 19 & 6819928 & NM_175498 & Pnma2 & Paraneoplastic antigen MA2 & 0.044 & -1.575 \\
\hline 20 & 6960931 & NM_001033962 & Ube3a & Ubiquitin protein ligase $\mathrm{E} 3 \mathrm{~A}$ & 0.016 & -1.579 \\
\hline 21 & 6971996 & NM_021302 & Stk32c & Serine/threonine kinase $32 \mathrm{C}$ & 0.029 & -1.587 \\
\hline 22 & 6764046 & BC126965 & Pcp4l1 & Purkinje cell protein 4 -like 1 & 0.029 & -1.589 \\
\hline 23 & 6819244 & NM_009947 & Cpne6 & Copine VI & 0.014 & -1.601 \\
\hline 28 & 6820282 & NM_172812 & $H \operatorname{tr} 2 a$ & 5-Hydroxytryptamine (serotonin) receptor $2 \mathrm{~A}$ & 0.010 & -1.625 \\
\hline 29 & 6760417 & NM_021306 & Ecel1 & Endothelin-converting enzyme-like 1 & 0.004 & -1.631 \\
\hline 30 & 6933072 & NM_009263 & Spp1 & Secreted phosphoprotein 1 & 0.047 & -1.633 \\
\hline 31 & 6994790 & NM_178737 & AW551984 & Expressed sequence AW551984 & 0.025 & -1.634 \\
\hline 32 & 6982725 & $\mathrm{BC} 111102$ & 4930431LO4Rik & RIKEN cDNA 4930431L04 gene & 0.032 & -1.636 \\
\hline 33 & 6762197 & NM_008795 & Pctk3 & PCTAIRE-motif protein kinase 3 & 0.029 & -1.638 \\
\hline 34 & 6805200 & NM_145451 & Gpx6 & Glutathione peroxidase 6 & 0.026 & -1.639 \\
\hline 35 & 6832276 & NM_172610 & Mpped 1 & Metallophosphoesterase domain containing 1 & 0.017 & -1.658 \\
\hline 36 & 6947131 & NM_028880 & Lrrtm1 & Leucine-rich repeat transmembrane neuronal 1 & 0.044 & -1.669 \\
\hline 37 & 6810961 & NM_033269 & Chrm3 & Cholinergic receptor, muscarinic 3 & 0.020 & -1.679 \\
\hline 38 & 6864813 & NM_011898 & Spry4 & Sprouty homologue 4 (Drosophila) & 0.022 & -1.687 \\
\hline 39 & 6750314 & NM_177164 & A830006F12Rik & RIKEN cDNA A830006F12 gene & 0.005 & -1.698 \\
\hline 47 & 6803891 & NM_178915 & Tmem179 & Transmembrane protein 179 & 0.005 & -1.788 \\
\hline 48 & 6988976 & NM_010077 & $\operatorname{Drd} 2$ & Dopamine receptor 2 & 0.017 & -1.794 \\
\hline 49 & 6815027 & NM_009027 & Rasgrf2 & RAS protein-specific guanine nucleotide-releasing factor 2 & 0.044 & -1.815 \\
\hline 50 & 6931355 & NM_011670 & Uchl1 & Ubiquitin C-terminal hydrolase L1 & 0.041 & -1.822 \\
\hline 51 & 6906620 & NM_011839 & Mab2112 & Mab-21-like 2 (C. elegans) & 0.031 & -1.979 \\
\hline 52 & 6862816 & NM_144946 & Neto1 & Neuropilin (NRP) and tolloid (TLL)-like 1 & 0.016 & -1.981 \\
\hline 53 & 6785684 & NM_010904 & Nefh & Neurofilament, heavy polypeptide & 0.043 & -2.088 \\
\hline 54 & 6894253 & NM_015730 & Chrna4 & Cholinergic receptor, nicotinic, $\alpha$-polypeptide 4 & 0.004 & -2.093 \\
\hline 55 & 6886908 & NM_013613 & $\mathrm{Nr} 4 \mathrm{a} 2$ & Nuclear receptor subfamily 4 , group $A$, member 2 & 0.028 & -2.148 \\
\hline 56 & 6844649 & NM_009215 & Sst & Somatostatin & 0.041 & -2.211 \\
\hline 57 & 6796691 & NM_010234 & Fos & FBJ osteosarcoma oncogene & 0.015 & -2.242 \\
\hline 58 & 6889978 & NM_010825 & Mrg1 & Myeloid ecotropic viral integration site-related gene 1 & 0.023 & -2.334 \\
\hline 59 & 6871062 & NM_153553 & Npas4 & Neuronal PAS domain protein 4 & 0.012 & -2.479 \\
\hline 60 & 6967593 & NM_176942 & Gabra5 & $\gamma$-Aminobutyric acid (GABA-A) receptor & 0.017 & -2.548 \\
\hline 61 & 6943974 & NM_009311 & $\operatorname{Tac1}$ & Tachykinin 1 & 0.013 & -2.615 \\
\hline 62 & 6833311 & NM_010444 & Nr4a1 & Nuclear receptor subfamily 4 , group $A$, member 1 & 0.016 & -2.741 \\
\hline 63 & 6788423 & NM_020492 & Glra1 & Glycine receptor, $\alpha-1$ subunit & 0.031 & -4.946 \\
\hline 64 & 6881459 & NM_029530 & 6330527006Rik & RIKEN cDNA 6330527006 gene & 0.010 & -6.616 \\
\hline
\end{tabular}

Differentially expressed genes with a fold change $\geq 1.5(P \leq 0.05)$ from Ube3 $a^{\mathrm{m}-/ \mathrm{p}+}$ mice compared with the wild-type littermates. List is shown in the order of the most upregulated, to the most downregulated gene (as shown by '-' sign). 
The most heavily represented downregulated genes in the $U b e 3 a^{\mathrm{m}-/ \mathrm{p}+}$ mice appear to encode receptors for neurogenic functions, such as neurotransmitter receptors (eg Glra1/3, Chrna4 and Drd2). Another substantial group of genes that were downregulated involves transcription regulation, functioning in neurogenesis and other physiological aspects (eg Mc1r, Nr4a2 and Npas).

\section{Pathway analysis}

Pathway analysis shows that the differentially expressed genes are implicated in three major pathways/networks including cell signaling, nervous system development and cell death. Fifteen genes are involved in the first network associated with cell signaling (Figure 1a), including Fgf7 and Nr4a2. In the brain, the orphan receptor Nr4a2 supports dopaminergic neurons to survive and differentiate. ${ }^{15}$ Twelve genes are associated with the nervous system development and functions (Figure 1b). Among them are Epha6, a tyrosine kinase receptor important for axon guidance, as well as Slc5a7, which encodes choline transporter responsible for proper choline uptake along the synapse. ${ }^{18,19}$ Eleven genes are associated with cellular development/ death (Figure 1c). Among them, downregulation of Mc1r and downregulation of Glral are two examples that show functional relevance to AS.

\section{Differential expression validation}

We have validated and confirmed the differential expression status of two genes per network described above, using semiquantitative reverse-transcription PCR (Figure 2a) and qRT-PCR in biological triplicates (Figure 2b). These validated genes (ie Fgf7, Glra1, Mclr, Nr4a2, Slc5a7 and Epha6) were chosen because their functions are relevant to the AS phenotype.

A recent report shows that Mc1r signaling induces the expression of Nr4a2. ${ }^{17}$ Because both mRNAs are downregulated in the AS mice, we extended our differential expression analysis to the protein level for these two genes. To determine if downregulation of Mc1r and Nr4a2 can be reflected at the protein levels, we performed Western blot comparing cerebellum total protein extract from wild-type and $U b e 3 a^{\mathrm{m}-/ \mathrm{p}+}$ mice. We found that both Mclr (Figure 2c) and Nr4a2 proteins (Figure 2d), like its relative transcript, are downregulated in the AS mouse.

\section{Ube3a knockdown in P19 cell line}

We were interested in finding out if knockdown of Ube3a in P19 cell line will lead to downregulation of $\mathrm{Mclr}$ and $\mathrm{Nr} 4 a 2$ similar to what we have observed in the Ube $3 a^{\mathrm{m}-/ \mathrm{p}+}$ mice. It is possible that the constitutive loss of Ube3a activity during mouse development may result in adaptive change in gene expression (to cope with loss of Ube3a activity), and thus many of the changes observed in the transcriptional level may represent indirect, rather than direct, consequences on loss of Ube3a activity.

To address this problem, we have generated an RNAi system with target sequence against $U b e 3 a$, in which immediate effect of loss of Ube3a activity on respective genes/proteins can be evaluated. The P19 cells transfected with shRNA expression plasmid show downregulation of Ube $3 a$ at both transcription and protein levels. There is a two-fold reduction in the Ube3a mRNA level after knockdown (Figure $3 a$ and $b$ ). Consistent with this result, Ube3a protein in P19 cells was reduced after transfection with the Ube3a shRNA expression plasmid as determined by Western blot analysis (Figure 3c). We then check for relative transcript expression of $\mathrm{Mcl} r$ and $\mathrm{Nr} 4 \mathrm{a} 2$ in the Ube3a knockdown and control cells. Semiquantitative reverse-transcription PCR (Figure 3a) and biological triplicates of qRT-PCR (Figure 3b) show that Mc1r and Nr4a2 mRNA levels decreased by 8.6- and 5.3-fold, respectively, in the Ube3a knockdown cells. These results suggest that functional Ube3a is perhaps required for Mclr and Nr4a2 gene expression.

\section{Overexpression of Ube3a in P19 cell line}

Because downregulation of $\mathrm{Mclr}$ and $\mathrm{Nr} 4 \mathrm{a} 2$ is observed in the Ube $3 \mathrm{a}^{\mathrm{m}-/ \mathrm{p}+}$ mice and Ube $3 a$ knockdown P19 cells, we were interested in determining if the levels of Mclr and Nr4a2 will be affected when Ube $3 a$ is overexpressed. We constructed an Ube3a expression vector, pUbe3aOE, and transfection of P19 cells with this plasmid resulted in higher level of Ube3a expression as determined by Western blot (Figure 4a). Subsequently, the mRNA level of Mc1r and Nr4a2 was determined using qRT-PCR. We observed a 5.9- and 10.9-fold increase in the mRNA level of Mclr and Nr4a2, respectively (Figure $4 \mathrm{~b}$ ).

\section{DISCUSSION}

Lack of functional maternal Ube3a expression in imprinted brain tissue can result in the accumulation of target proteins that are meant to be degraded through the ubiquitin proteosomal system, as well as dysregulation of genes expression due to the lack of the coactivation function of Ube3a. We used a genome-wide approach to detect differential genes expression between wild-type and Ube $3 \mathrm{a}^{\mathrm{m}-/ \mathrm{p}+}$ mice cerebellum. The mouse cerebellum was used because previous studies show that Ube3a is imprinted in the cerebellum and electrophysiology recording reveal abnormal oscillatory activity in the AS mice. ${ }^{5,20}$ In addition, the cerebellum controls motor movements, which most AS patient lack. ${ }^{3}$ P19 cells were used to investigate the knockdown/overexpression effect of Ube3a because these pluripotent embryonic cells can be induced to differentiate into neurons. ${ }^{21}$ In addition, neurons and skin are derived from the same lineage progenitor cells. $^{22}$

In this study, we have shown that Mclr is downregulated in AS mice at mRNA and protein levels. Mclr, a G-protein-coupled receptor, is widely studied in peripheral tissues, such as the skin, for its regulation of pigment production. ${ }^{23}$ OCA2, an autosomal recessive gene that is responsible for type 2 oculocutaneous albinism, is currently attributed toward the hypopigmentation phenotype seen in type I (deletion) AS patients as it lies within the AS deletion region along $15 \mathrm{q} 11-\mathrm{q} 13 .{ }^{24,25}$ However, this does not explain why other AS patients with UBE3A mutations, imprinting defect along 15q11-q13 or paternal uniparental disomy (type II-IV), which have intact OCA2, still show to a certain extent, the hypopigmentation phenotype. ${ }^{26}$ Gene expression in AS mouse cerebellum used in this study may be different from gene expression in other tissues such as the skin, even though neurons and skin are derived from the same lineage cells. ${ }^{22}$ However it would be interesting to determine if Ube3a mutation also causes downregulation of Mclr in the skin, resulting in the perturbation of normal pigment production seen in type II-IV patients. Co-deletion of OCA2 and UBE3A, which results in the decrease of Mclr, observed in type I deletion patients could perhaps lead to a synergistic effect, resulting in the stable and full-blown hypopigmentation phenotype.

In the brain, Mclr was shown to prevent inflammation, as well as to provide a neuroprotective effect on the brain cell population. ${ }^{16}$ On top of that, a recent report shows that Mclr signaling rapidly, yet transiently, induces transcription of the Nr4a subfamily receptors. ${ }^{17}$ The Nr4a subfamily receptors, Nr4a1/Nurr77, Nr4a2/Nurr1, and Nr4a3/NOR-1 are orphan receptors, well known for their close ligand-binding sites. ${ }^{27}$ We have shown that in the absence of maternal Ube3a in the brain, Nr4a2 transcript is reduced. Taken together, it is 

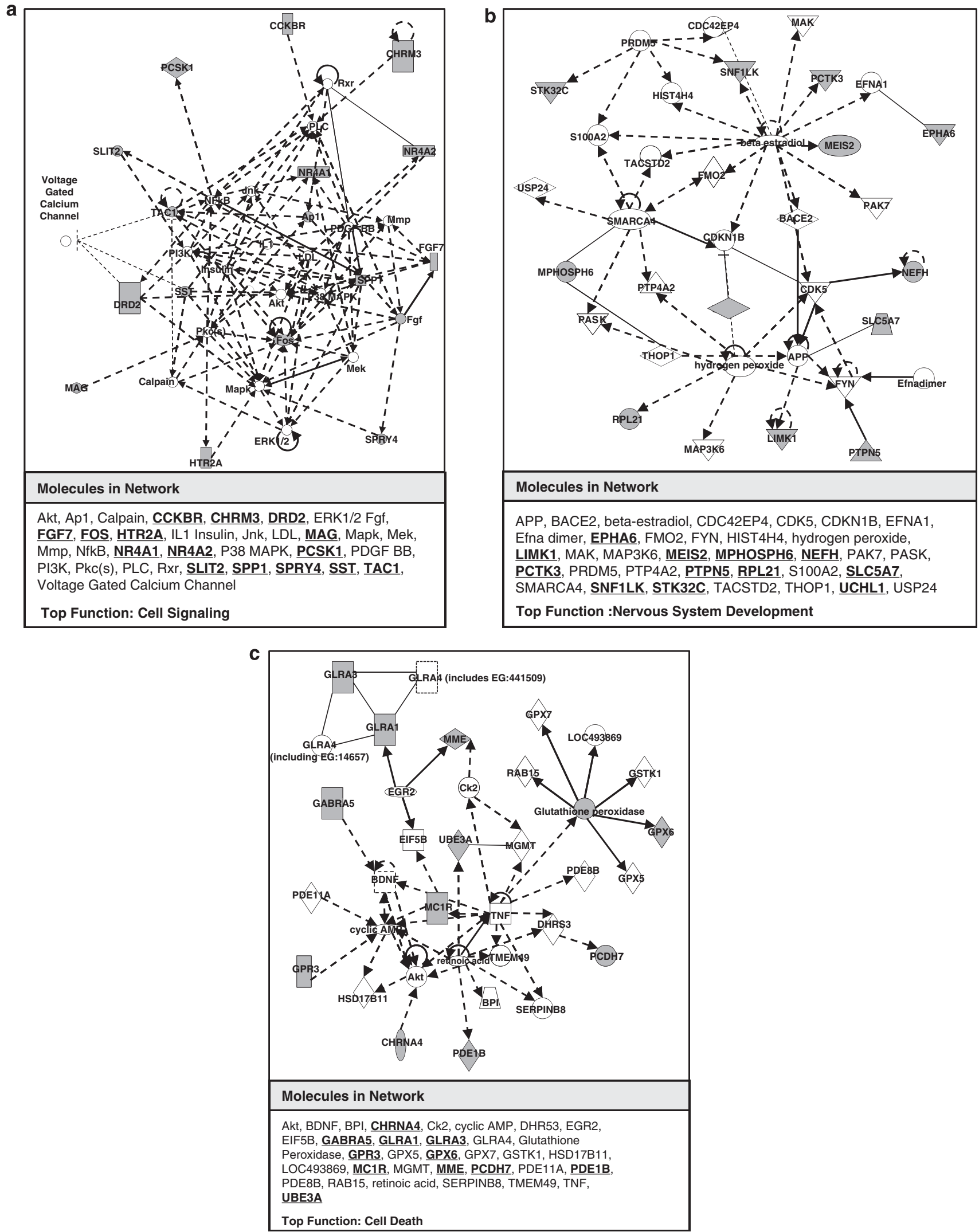

Figure 1 Pathway analysis on differentially expressed genes. (a) Network 1 is associated with cell signaling and involves 15 of our reported differentially expressed genes (in bold). Representative genes such as Fgf7 and Nr4a2 are qRT-PCR validated, which are up- and downregulated in the Ube3am-/p+ mice, respectively. (b) Network 2 is associated with nervous system development and involves 12 of our reported microarray hits. Epha6 and S/c5a7 in this network are qRT-PCR validated showing both downregulations in the Ube $3 a^{m-/ p+}$ mice. (c) Network 3 is associated with cell death and involves 11 genes, such as Glral and Mc1r. Both are down-regulated in the Ube3a $a^{\mathrm{m}-\mathrm{p}+}$ mice and validated using qRT-PCR. 
Up-Regulated in Mutant

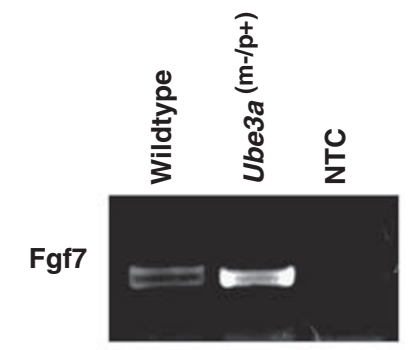

$\underline{\text { Down-Regulated in Mutant }}$
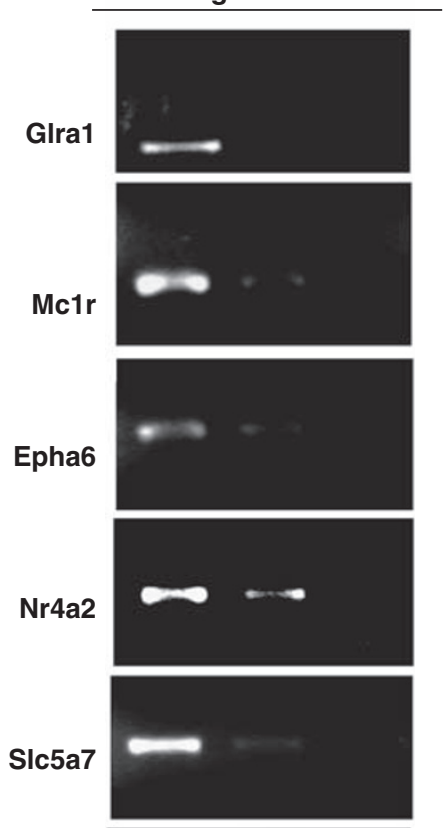

G3pdh

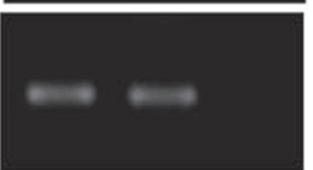

b

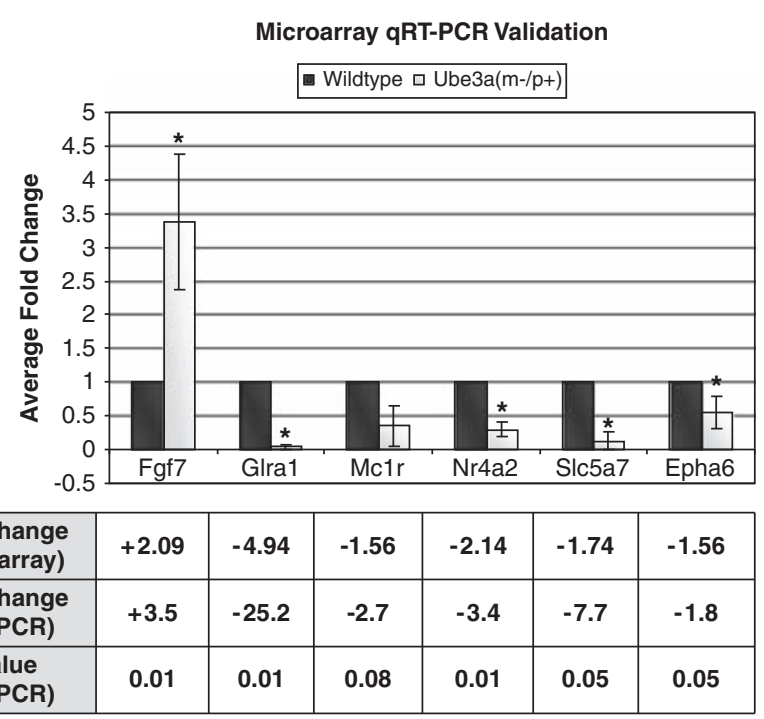

C
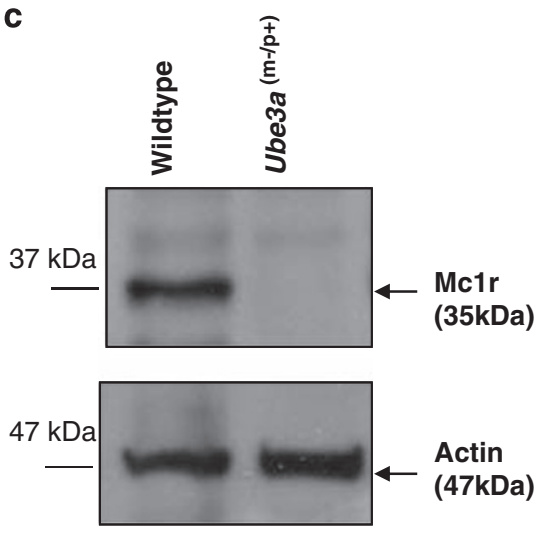

d

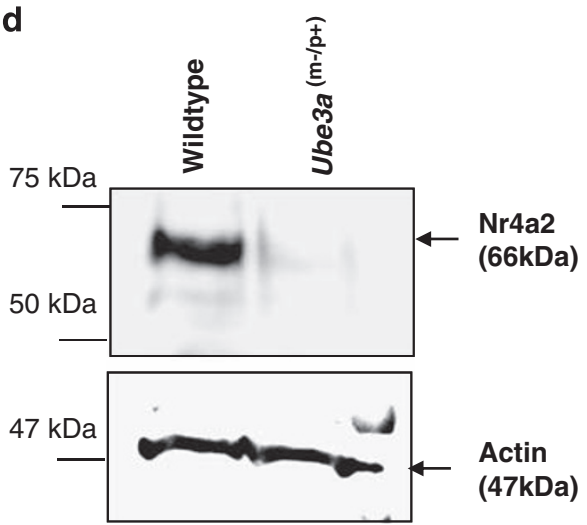

Figure 2 Semiquantitative reverse-transcription PCR, qRT-PCR and Western blot validation confirming on a selection of differentially expressed genes identified by microarray. (a) Semiquantitative reverse-transcription PCR validation: Fgf7 is upregulated in Ube3a ${ }^{\mathrm{m}-/ \mathrm{p}+}$ mice, whereas the rest of the genes, including Glra1, Mc1r, Nr4a2, Epha6 and S/c5a, are confirmed to be downregulated. NTC: no template control. (b) qRT-PCR validation showing the normalized mean fold change from the biological triplicates. The fold change is calculated using $\left.2^{- \text {(mean wild-type } \Delta C T-m e a n ~ U b e 3 a(m-/ p+) ~} \Delta C T\right)$; ' + ' and ' - ' represent upregulation and downregulation of transcript, respectively; ${ }^{*} P<0.05$. (c and d) Total protein $(10 \mu \mathrm{g})$ extracted from mouse cerebellum was analyzed by SDS-PAGE using 6\% acrylamide gel. Western blot analyses using antibody against Mc1r and Nr4a2 show that the $35 \mathrm{kDa}$ Mc1r (c) and the $66 \mathrm{kDa} \mathrm{Nr4a2}$ (d) proteins, respectively, are downregulated in the Ube3am-/p+ mice. $\beta$-Actin is used as endogenous internal control in the Western blot analyses.

conceivable that Ube3a might have a direct role in stimulating the synthesis of Mclr, which in turn, regulates Nr4a2 gene expression.

Reducing the expression of $\mathrm{Nr} 4 \mathrm{a} 2$ in the brain might explain certain AS phenotype, including poor learning/memory, and motor incoordination. Nr4a2 knockdown in rat hippocampus was reported to affect spatial discrimination, learning and memory. ${ }^{28}$ In the AS mouse model, where Nr4a2 is downregulated, the mice show severe long-term potentiation and learning impairment. ${ }^{9}$ In contrast, $\mathrm{Nr} 4 \mathrm{a} 2$ mRNA expression was found to be increased during learning in the rat models. ${ }^{29}$ In a recent report, $\mathrm{Nr} 4 \mathrm{a} 2$ has been shown to interact with Wnt signaling via $\beta$-catenin in the establishment and development of the nervous system. ${ }^{30}$ More importantly, Nr4a2 was reported to be critical for induction and survival of dopaminergic neurons. ${ }^{15} \mathrm{Nr} 4 \mathrm{a} 2^{+/-}$ mice appear normal at birth, but show motor abnormality as a result of reduce numbers of dopaminergic neurons. ${ }^{31}$ Hence, the motor dysfunction observed in AS patients as a result of loss of maternal Ube3a could possibly be related to the decrease levels of $\mathrm{Nr} 4 \mathrm{a} 2$, which mediates the induction and survival of dopaminergic neurons. ${ }^{15}$

In addition, we have identified many neurotransmitter receptors that are differentially expressed in the AS mice, including glycine receptor (Glra1), $\gamma$-aminobutyric acid receptor (Gabra5) and cholinergic receptor (Chrna4). The localization of UBE3A ${ }^{\mathrm{YFP}}$ fusion gene at the pre/postsynaptic regions of cultured hippocampal neurons led to the speculation that UBE3A may directly regulate the development and/or synaptic functions. ${ }^{8}$ Downregulation of neurotransmitter receptors could affect proper neuro-signal transduction and normal neuronal and motor functions. For example, mutations in Glra1 result 


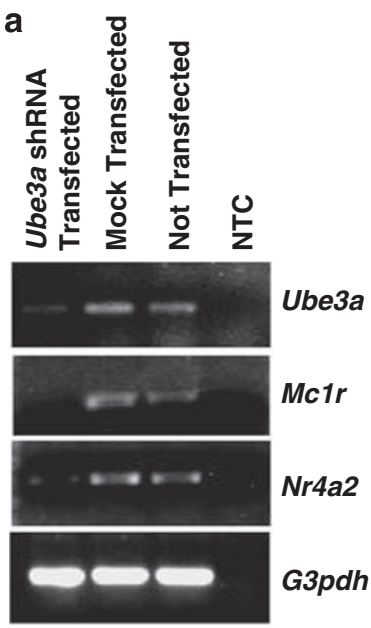

b
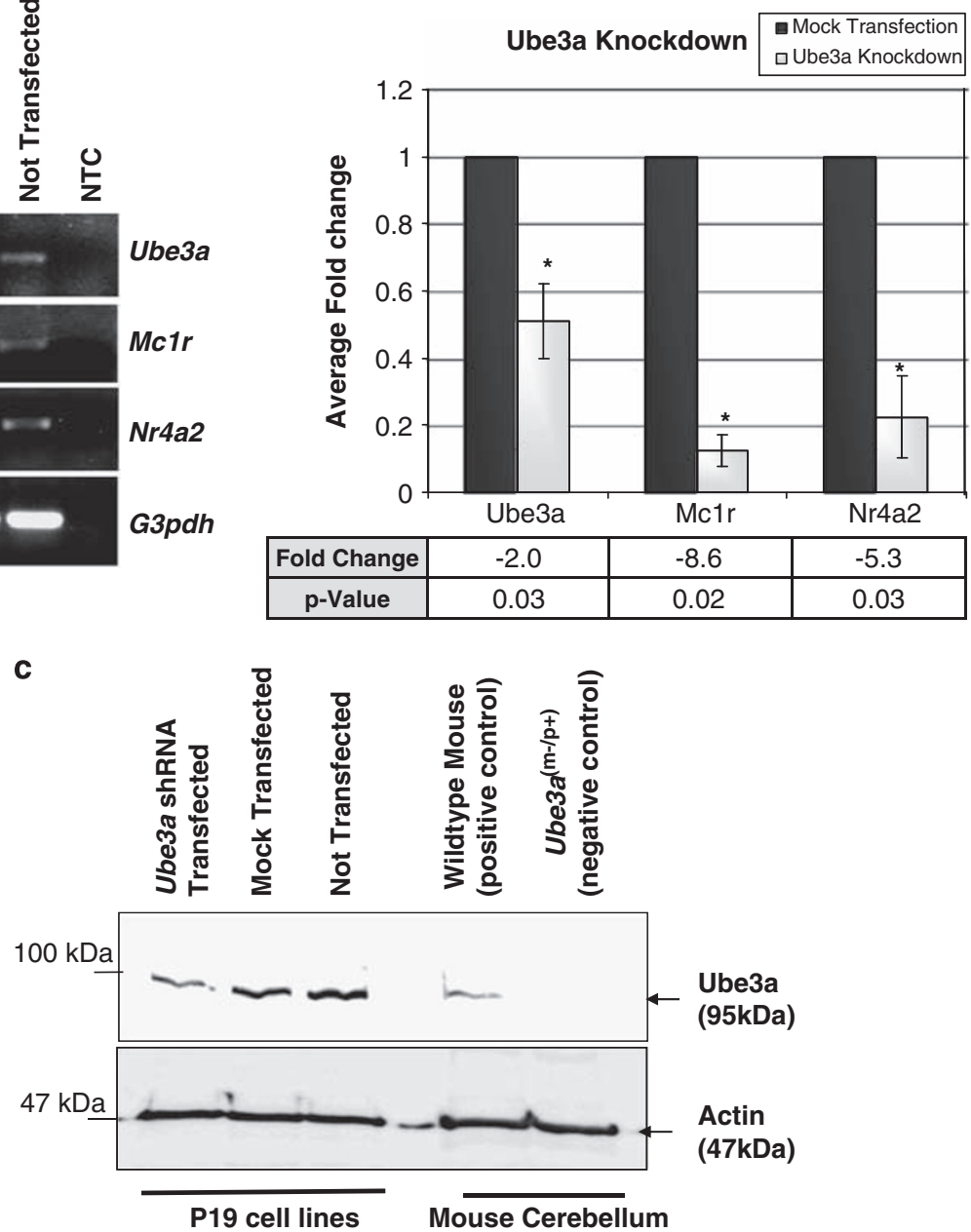

Figure 3 Validation of downregulation of $M c 1 r$ and Nr4a2 by shRNA-mediated knockdown of Ube3a in P19 cells. (a) Semiquantitative reverse-transcription PCR showing the downexpression of Ube3a, Mclr and Nr4a2 transcript in the Ube3a shRNA-transfected cells. NTC: no template control. (b) Biological triplicates of qRT-PCR analyses showing the normalized mean fold change. '-' represents a downregulation in the Ube3a shRNA-transfected cells; * $P<0.05$. (c) Total protein $(10 \mu \mathrm{g})$ extracted from the Ube3a shRNA-transfected and control P19 cells was analyzed by SDS-PAGE using 6\% acrylamide gel. Western blot analyses using antibody against Ube3a show the knockdown of the $95 \mathrm{kDa}$ Ube3a on transfection with the Ube3a shRNA plasmid (pUbe3aKD). Total protein extracted from wild-type and the Ube $3 a^{\mathrm{m}-\mathrm{p}+}$ mice cerebellum was used as positive and negative control in the same Western blot analyses. $\beta$-Actin is used as endogenous internal control in the Western blot analysis.

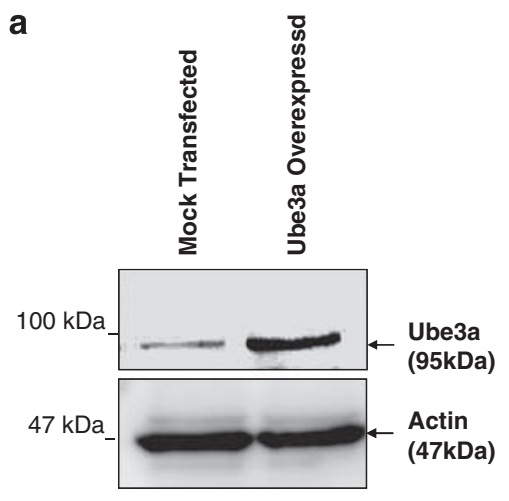

b

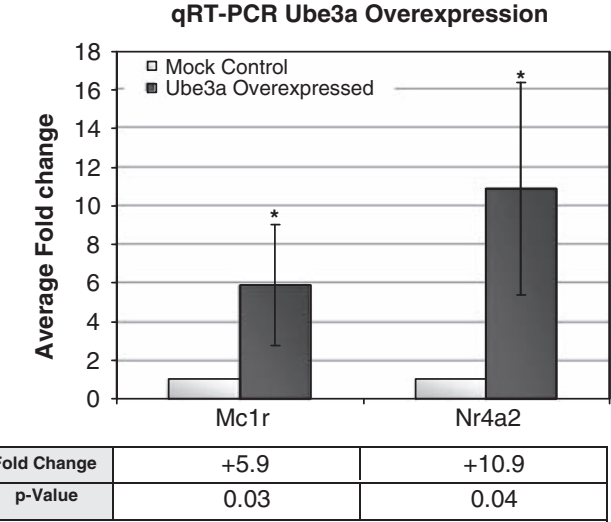

Figure 4 Ube3a overexpression results in an upregulation of Mc1r and Nr4a2. (a) Total protein (10 $\mu \mathrm{g}$ ) extracted from P19 cells transfected with the Ube3a expression plasmid (pUbe3aOE) and control cells was analyzed by SDS-PAGE using $6 \%$ acrylamide gel. Western blot analyses using antibody against Ube3a show the increase protein level of the $95 \mathrm{kDa}$ Ube3a on overexpression of Ube3a. $\beta$-Actin is used as endogenous internal control in the Western blot analysis. (b) Biological triplicates of qRT-PCR analyses showing the normalized mean fold change of Mc1r and Nr4a2 on Ube3a overexpression in P19 cells. ' + ' represents an upregulation in the Ube3a-overexpressed cells; ${ }^{*} P<0.05$. 
in hyperekplexia, where patients show 'drop seizure' phenotype. ${ }^{32}$ These symptoms show overlapping phenotype to the AS, creating a possibility that the cause of some of the AS phenotype, such as seizure, might be associated with lack of Glral expression. The reason why Gabra5 is downregulated in Ube3a-deficient mouse is unclear. Besides direct or indirect mechanisms involving the loss of the coactivator and/or ubiquitin ligase functions of Ube3a, it is possible that a chromatin structure alteration, or the loss of a positive regulatory element caused by Ube3a knockout may lead to downregulation of Gabra5, given that Gabra5 is located adjacent to Ube3a in mouse (7c) and human (15q12). A similar effect is seen for upregulation of Irak1 in the Mecp2-deficient mouse model. ${ }^{33,34}$

Altogether, we had performed a genome-wide gene expression profiling of $U b e 3 a^{\mathrm{m}-/ \mathrm{p}+}$ mice with the intention of identifying genes that are affected in the absence of functional Ube3a. We have observed 64 genes that are differentially expressed in the AS mice. These genes fit into three major networks associated with cell signaling, nervous system development and cell death. We validated the expression of six representative genes (Fgf7, Glra1, Mclr, Nr4a2, Epha6, and Slc5a7) using qRT-PCR. Using an shRNA knockdown system, we have shown that Ube3a knockdown causes downregulation of Mc1r and Nr4a2 in the embryonic P19 cell, whereas overexpression of Ube3a results in upregulation of $\mathrm{Mc1r}$ and $\mathrm{Nr} 4 \mathrm{a} 2$, suggesting that Ube3a is involved in regulating their expression. These results can provide a step forward toward a better understanding of AS development and narrows down some critical genes affected in the event of Ube3a mutations that might contribute to the onset of the disease.

\section{CONFLICT OF INTEREST}

The authors declare no conflict of interest.

\section{ACKNOWLEDGEMENTS}

This work was supported by the Academic Research Fund (M52080025) from Nanyang Technological University awarded to Ken-Shiung Chen.

1 Clayton-Smith J, Driscoll DJ, Waters MF et al: Difference in methylation patterns within the D15s9 region of chromosome $15 q 11-13$ in 1st cousins with Angelman syndrome and Prader-Willi syndrome. Am J Med Genet 1993; 47: 683-686.

2 Thomson AK, Glasson EJ, Bittles AH: A long-term population-based clinical and morbidity profile of Angelman syndrome in Western Australia: 1953-2003. Disabil Rehabil 2006; 28: 299-305.

3 Williams CA, Beaudet AL, Clayton-Smith J et al: Angelman syndrome 2005: updated consensus for diagnostic criteria. Am J Med Genet A 2006; 140A: 413-418.

4 Kishino T, Lalande M, Wagstaff J: UBE3A/E6-AP mutations cause Angelman syndrome. Nat Genet 1997; 15: 70-73.

5 Albrecht U, Sutcliffe JS, Cattanach BM et al: Imprinted expression of the murine Angelman syndrome gene, Ube3a, in hippocampal and Purkinje neurons. Nat Genet 1997; 17: 75-78

6 Scheffner M, Huibregtse JM, Vierstra RD, Howley PM: The Hpv-16 E6 and E6-Ap complex functions as a ubiquitin-protein ligase in the ubiquitination of P53. Cell 1993; 75: 495-505.

7 Nawaz Z, Lonard DH, Smith CL et al: The Angelman syndrome-associated protein, E6-AP, is a coactivator for the nuclear hormone receptor superfamily. Mol Cell Biol 1999; 19: 1182-1189.
8 Dindot SV, Antalffy BA, Bhattacharjee MB, Beaudet AL: The Angelman syndrome ubiquitin ligase localizes to the synapse and nucleus, and maternal deficiency results in abnormal dendritic spine morphology. Hum Mol Genet 2008; 17: 111-118.

9 Jiang $\mathrm{YH}$, Armstrong $\mathrm{D}$, Albrecht $\mathrm{U}$ et al: Mutation of the Angelman ubiquitin ligase in mice causes increased cytoplasmic p53 and deficits of contextual learning and long-term potentiation. Neuron 1998; 21: 799-811.

10 Lu YB, Wang F, Li Y, Ferris J, Lee JA, Gao FB: The Drosophila homologue of the Angelman syndrome ubiquitin ligase regulates the formation of terminal dendritic branches. Hum Mol Genet 2009; 18: 454-462.

11 Huibregtse JM, Scheffner M, Howley PM: Localization of the E6-AP regions that direct human papillomavirus E6 binding, association with P53, and ubiquitination of associated proteins. Mol Cell Biol 1993; 13: 4918-4927.

12 Reiter LT, Seagroves TN, Bowers M, Bier E: Expression of the Rho-GEF Pbl/ECT2 is regulated by the UBE3A E3 ubiquitin ligase. Hum Mol Genet 2006; 15: 2825-2835

13 Zheng L, Ding HR, Lu ZM et al: E3 ubiquitin ligase E6AP-mediated TSC2 turnover in the presence and absence of HPV16 E6. Genes Cells 2008; 13: 285-294.

14 Mishra A, Godavarthi SK, Maheshwari M, Goswami A, Jana NR: The ubiquitin ligase E6-AP is induced and recruited to aggresomes in response to proteasome inhibition and may be involved in the ubiquitination of Hsp70-bound misfolded proteins. J Biol Chem 2009; 284: 10537-10545.

15 Saucedo-Cardenas O, Quintana-Hau JD, Le WD et al: Nurr 1 is essential for the induction of the dopaminergic phenotype and the survival of ventral mesencephalic late dopaminergic precursor neurons. Proc Natl Acad Sci USA 1998; 95: 4013-4018.

16 Catania A: Neuroprotective actions of melanocortins: a therapeutic opportunity. Trends Neurosci 2008; 31: 353-360.

17 Smith AG, Luk N, Newton RA, Roberts DW, Sturm RA, Muscat GEO: Melanocortin-1 receptor signaling markedly induces the expression of the NR4A nuclear receptor subgroup in melanocytic cells. J Biol Chem 2008; 283: 12564-12570.

18 Apparsundaram S, Ferguson SM, George AL, Blakely RD: Molecular cloning of a human, hemicholinium-3-sensitive choline transporter. Biochem Biophys Res Commun 2000; 276: 862-867.

19 Yue Y, Chen ZY, Gale NW et al: Mistargeting hippocampal axons by expression of a truncated Eph receptor. Proc Natl Acad Sci USA 2002; 99: 10777-10782.

20 Cheron G, Servais L, Wagstaff J, Dan B: Fast cerebellar oscillation associated with ataxia in a mouse model of Angelman syndrome. Neuroscience 2005; 130: 631-637.

21 Jonesvilleneuve EMV, McBurney MW, Rogers KA, Kalnins VI: Retinoic acid induces embryonal carcinoma cells to differentiate into neurons and gial cells. J Cell Biol 1982; 94: 253-262.

22 Baroffio A, Dupin E, Ledouarin NM: Common precursors for neural and mesectodermal derivatives in the cephalic neural crest. Development 1991; 112: 301-305.

23 Luger TA, Paus R, Lipton JM, Slominski AT (eds):: The melanocortin-1 receptor and human pigmentation. Conference on Cutaneous Neuroimmunomodulation - The Proopiomelanocortin System; 11-13 Sep; Munster, Germany.

24 Oetting WS, King RA: Molecular basis of albinism: mutations and polymorphisms of pigmentation genes associated with albinism. Hum Mutat 1999; 13: 99-115.

25 Fridman C, Hosomi N, Varela MC, Souza AH, Fukai K, Koiffmann CP: Angelman syndrome associated with oculocutaneous albinism due to an intragenic deletion of the P gene. Am J Med Genet A 2003; 119A: 180-183.

26 Lossie AC, Whitney MM, Amidon D et al: Distinct phenotypes distinguish the molecular classes of Angelman syndrome. J Med Genet 2001; 38: 834-845.

27 Maxwell MA, Muscat GE: The NR4A subgroup: immediate early response genes with pleiotropic physiological roles. Nucl Recept Signal 2006; 4: e002.

28 Colon-Cesario WI, Martinez-Montemayor MM, Morales S et al: Knockdown of Nurr1 in the rat hippocampus: implications to spatial discrimination learning and memory. Learn Mem 2006; 13: 734-744.

29 de Ortiz SP, Maldonado-Vlaar CS, Carrasquillo Y: Hippocampal expression of the orphan nuclear receptor gene hzf-3/nurr1 during spatial discrimination learning. Neurobiol Learn Mem 2000; 74: 161-178.

30 Kitagawa $\mathrm{H}$, Ray WJ, Glantschnig $\mathrm{H}$ et al: A regulatory circuit mediating convergence between Nurr1 transcriptional regulation and Wnt signaling. Mol Cell Biol 2007; 27: 7486-7496.

31 Jiang $\mathrm{CT}$, Wan $\mathrm{XH}$, He Y, Pan TH, Jankovic J, Le WD: Age-dependent dopaminergic dysfunction in Nurr1 knockout mice. Exp Neurol 2005; 191: 154-162.

32 Kirstein L, Silfverskiold BP: A family with emotionally precipitated drop seizures. Acta Psychiatr Neurol 1958; 33: 471-476.

33 Chahrour M, Jung SY, Shaw C et al: MeCP2, a key contributor to neurological disease, activates and represses transcription. Science 2008; 320: 1224-1229.

34 Urdinguio RG, Lopez-Serra L, Lopez-Nieva P et al: Mecp2-null mice provide new neuronal targets for Rett syndrome. PLoS One 2008; 3: e3669. 